\title{
Sesquiterpenoids from the Rhizomes of Homalomena occulta
}

\author{
Jun-Li Yang $\cdot$ Ya-Min Zhao $\cdot$ Yan-Ping Shi
}

Received: 22 May 2016/Accepted: 23 June 2016/Published online: 5 July 2016

(C) The Author(s) 2016. This article is published with open access at Springerlink.com

\begin{abstract}
Naturally occurring oplopanane sesquiterpenoids are rarely reported. A phytochemical investigation on the rhizomes of Homalomena occulta (Lours) has resulted in the discovery of six oplopanane sesquiterpenoids (1-6), including four new (1-4) and one 3,5-seco-oplopanane (6), together with three previously reported sesquiterpenoids (7-9). In addition three new oplopananes (2a-4a) were also obtained by chemical transformation. All structures of these sesquiterpenoids were established based on the comprehensive spectroscopic analyses, including NMR, MS, and IR, and comparing with the literatures.
\end{abstract}

\section{Graphical Abstract}

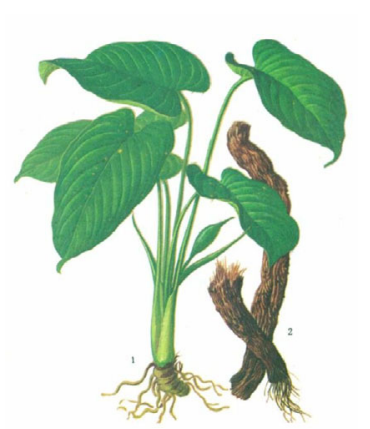

Homalomena occulta

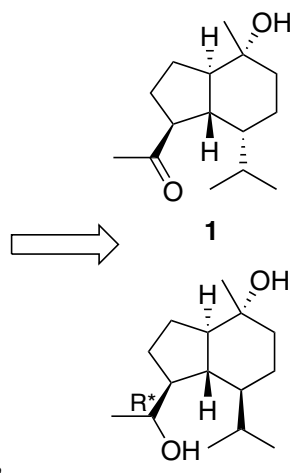

3

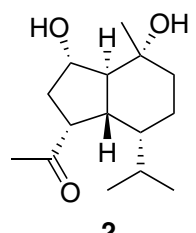

2

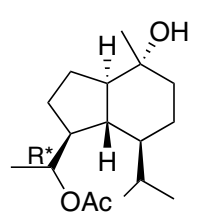

3a

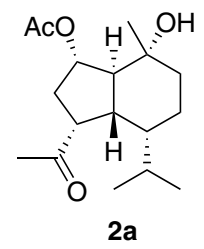

$2 a$
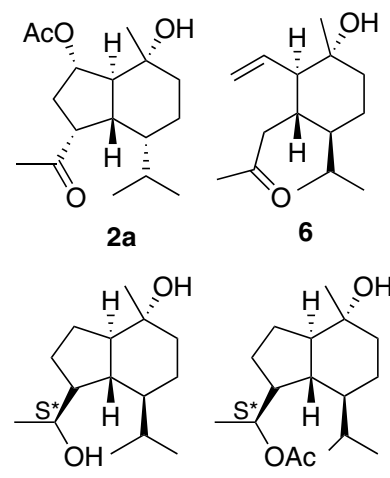

4

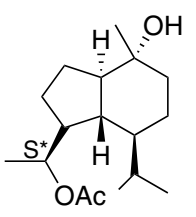

$4 \mathbf{a}$

Keywords Homalomena occulta $\cdot$ Sesquiterpenoids · Oplopananes

Electronic supplementary material The online version of this article (doi:10.1007/s13659-016-0104-8) contains supplementary material, which is available to authorized users.

J.-L. Yang · Y.-P. Shi $(\bowtie)$

Key Laboratory of Chemistry of Northwestern Plant Resources of CAS and Key Laboratory for Natural Medicine of Gansu Province, Lanzhou Institute of Chemical Physics, Chinese Academy of Sciences, Lanzhou 730000, People's Republic of China

e-mail: shiyp@licp.cas.cn

Y.-M. Zhao · Y.-P. Shi

State Key Laboratory of Applied Organic Chemistry, Lanzhou University, Lanzhou 730000, People's Republic of China

\section{Introduction}

With its rich cultural heritage and biodiversity, traditional Chinese medicine (TCM) has potential as a source for the discovery of structurally novel bioactive compounds. During the last twenty years, considerable efforts have been dedicated towards the exploration of the natural product chemistry of TCM. Homalomena occulta (Lour.) was officially listed in the Chinese Pharmacopoeia (named Qian-nian-jian) [1], and was found to occur in the tropical and sub-tropical areas of Asia and America. The plant has 
been used for the treatment of rheumatoid arthritis, trengthening tendons and bones, and invigorating the kidney and liver [2, 3]. Recent reports of $H$. occulta have also shown that the species is one of the most prolific sources of compounds with new structures [2-6].

Our research group has considerably focused on phytochemical investigations of TCM [7-10]. As part of our continuing efforts to obtain novel compounds with exquisite structural architectures, we initiated a chemical investigation of $H$. occulta, which has thus far led to the isolation and structural elucidation of six oplopanane sesquiterpenoids (1-6), including four new (1-4) and one 3,5-seco-oplopanane (6), together with three previously reported sesquiterpenoids (7-9).

\section{Results and Discussion}

By means of diverse chromatographic methods, including silica gel and LH-20, four new oplopanane sesquiterpenoids (1-4) (Fig. 1) have been purified from an $88 \%$ ethanol/water extract of the rhizomes of $H$. occulta.

Compound 1 was isolated as an optically active colorless oil with $[\alpha]_{D}^{20}-54\left(c 0.07, \mathrm{CHCl}_{3}\right)$. Its molecular formula was analyzed as same as oplopanone (5) [11], i.e. $\mathrm{C}_{15} \mathrm{H}_{26} \mathrm{O}_{2}$, based on a pseudomolecular ion peak at $m / z$ 256.2272 $\left[\mathrm{M}+\mathrm{NH}_{4}\right]^{+}$(calcd. 256.2271) in the positive HRESIMS spectrum, indicating three degrees of unsaturation. The IR absorptions suggested the existence of hydroxy $\left(3382 \mathrm{~cm}^{-1}\right)$ and ketone $\left(1705 \mathrm{~cm}^{-1}\right)$ functionalities. The ${ }^{13} \mathrm{C}$ NMR spectra (Table 1 ) showed the presence of one oxygenated quaternary carbon $\left(\delta_{\mathrm{C}} 73.2\right)$ and one ketone carbon $\left(\delta_{\mathrm{C}} 212.3\right)$. Overall NMR pattern, together with the established molecular formula, showed

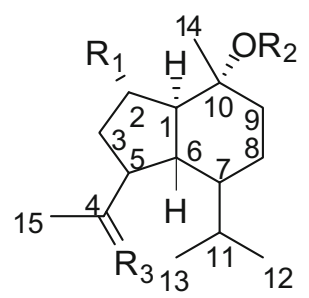

$15 \alpha \mathrm{H}, 7 \beta \mathrm{H}, \mathrm{R}_{1}=\mathrm{R}_{2}=\mathrm{H}, \mathrm{R}_{3}=\mathrm{O}$

$25 \beta \mathrm{H}, 7 \beta \mathrm{H}, \mathrm{R}_{1}=\mathrm{OH}, \mathrm{R}_{2}=\mathrm{H}, \mathrm{R}_{3}=\mathrm{O}$

2a $5 \beta \mathrm{H}, 7 \beta \mathrm{H}, \mathrm{R}_{1}=\mathrm{OAc}, \mathrm{R}_{2}=\mathrm{H}, \mathrm{R}_{3}=\mathrm{O}$

3, $45 \alpha \mathrm{H}, 7 \alpha \mathrm{H}, \mathrm{R}_{1}=\mathrm{R}_{2}=\mathrm{H}, \mathrm{R}_{3}=\mathrm{H}, \mathrm{OH}$

3a, 4a $5 \alpha H, 7 \alpha H, R_{1}=R_{2}=H, R_{3}=H$, OAc

$5 \quad 5 \alpha H, 7 \alpha H, R_{1}=R_{2}=H, R_{3}=0$

close resemblance to those of oplopanone (5) [11], which indicated that compounds $\mathbf{1}$ and $\mathbf{5}$ were possibly epimers of each other. The large coupling constants of $J_{\mathrm{H}-1, \mathrm{H}-6}$ $(12.0 \mathrm{~Hz})$ and $J_{\mathrm{H}-5, \mathrm{H}-6}(10.4 \mathrm{~Hz})$ indicated the trans relationship from $\mathrm{H}-1$ to $\mathrm{H}-6$, and from $\mathrm{H}-5$ to $\mathrm{H}-6$, while the small coupling constants of $J_{\mathrm{H}-6, \mathrm{H}-7}(4.0 \mathrm{~Hz})$ suggested $\mathrm{H}-7$ as $\beta$-oriented. A molecular modeling study based on no NOE correlation from $\mathrm{H}-1$ to $\mathrm{H}_{3}-14$ was used to determine the $\beta$-oriented Me-14. Finally, compound 1 was 6-epioplopanone.

Compound 2, obtained as an optically active colorless oil with $[\alpha]_{D}^{20}+20\left(c 0.1, \mathrm{CHCl}_{3}\right)$, was assigned a molecular formula $\mathrm{C}_{15} \mathrm{H}_{26} \mathrm{O}_{3}$ as analyzed from a pseudomolecular ion at $m / z$ 277.1779 $[\mathrm{M}+\mathrm{Na}]^{+}$(calcd. 277.1774) in its positive HRESIMS, indicative of three degrees of unsaturation. Its IR spectrum showed absorptions for hydroxy $\left(3392 \mathrm{~cm}^{-1}\right)$ and ketone $\left(1704 \mathrm{~cm}^{-1}\right)$ groups. A spectroscopic analogy of $\mathbf{2}$ with $\mathbf{1}$ indicated that compound $\mathbf{2}$ was an oplopanane-type sesquiterpenoid. The significant variations of $\mathbf{1}$ and $\mathbf{2}$ in their ${ }^{1} \mathrm{H}$ NMR spectra were the disappearance of methylene signals of $\mathrm{H}_{2}-2\left(\delta_{\mathrm{H}} 1.90 / 1.42\right)$ in 1 and the appearance of a multiplet at $\delta_{\mathrm{H}} 4.10$ in 2 . These changes could be explained by the presence of a hydroxy group at $\mathrm{C}-2$ in compound $\mathbf{2}$, which was further elucidated from an $\mathrm{HMBC}$ experiment. In confirmation of the relative configuration, $\mathbf{2}$ gave a 2-monoacetate (2a) (Table 2) after overnight treatment with $\mathrm{Ac}_{2} \mathrm{O} / \mathrm{Py}$ (Sect. 3). As in $\mathbf{2 a}$, the coupling constants $J_{1,6}=12.4 \mathrm{~Hz}, \quad J_{1,2}=9.2 \mathrm{~Hz}$, $J_{5,6}=2.4 \mathrm{~Hz}$, and $J_{6,7}=8.9 \mathrm{~Hz}$ allowed the placement of H-5, H-6, and $\mathrm{H}-7$ on $\alpha$-orientation, and $\mathrm{H}-1$ and $\mathrm{OH}-2$ on $\alpha$-orientation. The NOE correlations of $\mathrm{H}_{3}-14$ with $\mathrm{H}-2 / \mathrm{H}$ 6 , and $\mathrm{H}-5$ with $\mathrm{H}-2 / \mathrm{H}-7$ were used to determine $\mathrm{H}_{3}-14$ as $\beta$-oriented. Thus, the structure of $\mathbf{2}$ was established as 5,7diepi-2 $\alpha$-hydroxyoplopanone.
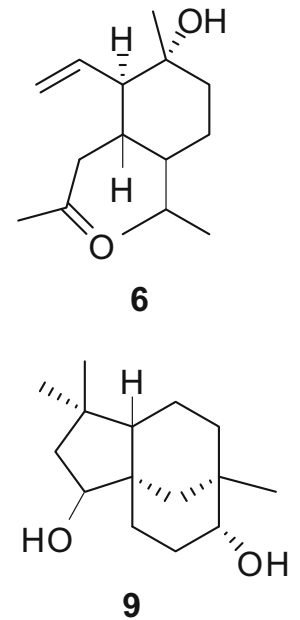

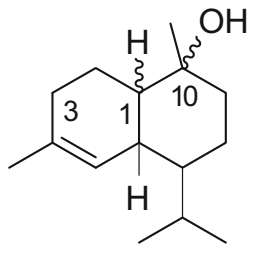

$71 \beta \mathrm{H}, 10 \beta \mathrm{OH}$

$81 \alpha \mathrm{H}, 10 \alpha \mathrm{OH}$

Fig. 1 Molecular structures of sesquiterpenoids 1-9 
Table 1 NMR spectroscopic data for compound 1 in $\mathrm{CDCl}_{3}$

\begin{tabular}{|c|c|c|c|c|c|}
\hline Position & $\delta_{\mathrm{H}}(J$ in $\mathrm{Hz})$ & $\delta_{\mathrm{C}}$ & Position & $\delta_{\mathrm{H}}(J$ in $\mathrm{Hz})$ & $\delta_{\mathrm{C}}$ \\
\hline \multirow[t]{2}{*}{1} & 2.07, ddd $(6.8,12.0,12.0)$ & $52.5(\mathrm{~d})$ & $\mathbf{8 a}$ & $1.55, \mathrm{~m}$ & $23.0(\mathrm{t})$ \\
\hline & & & $\mathbf{8 b}$ & $1.01, \mathrm{dd}(3.2,13.6)$ & \\
\hline $2 \mathbf{a}$ & $1.90, \mathrm{~m}$ & $24.7(\mathrm{t})$ & $9 a$ & $1.77, \mathrm{dt}(3.2,12.4)$ & $42.2(\mathrm{t})$ \\
\hline $2 \mathbf{b}$ & $1.42, \mathrm{~m}$ & & $9 b$ & $1.40, \mathrm{~m}$ & \\
\hline $3 \mathbf{a}$ & $1.94, \mathrm{dt}(2.8,6.0)$ & $27.5(\mathrm{t})$ & 10 & & $73.2(\mathrm{~s})$ \\
\hline $3 \mathbf{b}$ & $1.58, \mathrm{~m}$ & & & & \\
\hline 4 & & $212.3(\mathrm{~s})$ & 11 & $1.67, \mathrm{~m}$ & $29.2(\mathrm{~d})$ \\
\hline \multirow[t]{2}{*}{5} & 3.12, ddd $(3.2,10.4,10.4)$ & $50.5(\mathrm{~d})$ & 12 and 13 & $0.78, \mathrm{~d}(7.2)$ & $15.8(\mathrm{q})$ \\
\hline & & & & $0.88, \mathrm{~d}(7.2)$ & $21.3(\mathrm{q})$ \\
\hline 6 & 1.35 , ddd $(4.0,10.4,12.0)$ & 49.1 (d) & 14 & $1.09, \mathrm{~s}$ & $19.4(q)$ \\
\hline 7 & $1.59, \mathrm{~m}$ & 42.7 (d) & 15 & $2.15, \mathrm{~s}$ & $31.5(\mathrm{q})$ \\
\hline
\end{tabular}

Table 2 NMR spectroscopic data for compounds $\mathbf{2}, \mathbf{2 a}$, and $\mathbf{3}$ in $\mathrm{CDCl}_{3}$

\begin{tabular}{|c|c|c|c|c|c|c|}
\hline \multirow[t]{2}{*}{ Position } & \multicolumn{2}{|l|}{2} & \multicolumn{2}{|l|}{$2 \mathbf{a}$} & \multicolumn{2}{|l|}{3} \\
\hline & $\delta_{\mathrm{H}}(J$ in $\mathrm{Hz})$ & $\delta_{\mathrm{C}}$ & $\delta_{\mathrm{H}}(J$ in $\mathrm{Hz})$ & $\delta_{\mathrm{C}}$ & $\delta_{\mathrm{H}}(J$ in $\mathrm{Hz})$ & $\delta_{\mathrm{C}}$ \\
\hline 1 & $1.50, \mathrm{~m}$ & $60.4(d)$ & $1.75, \mathrm{dd}(9.2,12.4)$ & $59.9(\mathrm{~d})$ & 1.42 , dt $(4.8,11.6)$ & $57.5(\mathrm{~d})$ \\
\hline $2 \mathbf{a}$ & $4.10, \mathrm{~m}$ & $72.2(\mathrm{~d})$ & 5.08, ddd $(5.6,8.8,9.2)$ & 74.0 (d) & $1.78, \mathrm{dt}(4.8,12.4)$ & $25.4(\mathrm{t})$ \\
\hline $2 \mathbf{b}$ & & & & & $1.18, \mathrm{~m}$ & \\
\hline $\mathbf{3 a}$ & $1.82, \mathrm{~m}$ & $36.9(\mathrm{t})$ & $2.08, \mathrm{~m}$ & $35.8(\mathrm{t})$ & $1.75, \mathrm{~m}$ & $22.5(\mathrm{t})$ \\
\hline $3 \mathbf{b}$ & $1.82, \mathrm{~m}$ & & $1.93, \mathrm{~m}$ & & $1.58, \mathrm{~m}$ & \\
\hline 4 & & $210.9(\mathrm{~s})$ & & $210.0(\mathrm{~s})$ & $3.98, \mathrm{q}(6.4)$ & $68.6(d)$ \\
\hline 5 & $1.75, \mathrm{~m}$ & $42.2(\mathrm{~d})$ & 2.79, ddd $(2.4,8.0,10.0)$ & 44.1 (d) & $1.72, \mathrm{~m}$ & $48.9(\mathrm{~d})$ \\
\hline 6 & $2.73, \mathrm{~m}$ & $52.6(d)$ & 1.86, ddd $(2.4,8.9,12.4)$ & $53.2(\mathrm{~d})$ & $1.33, \mathrm{~m}$ & $44.6(d)$ \\
\hline 7 & $1.03, \mathrm{~m}$ & 49.5 (d) & $1.15, \mathrm{~m}$ & 49.7 (d) & $1.18, \mathrm{~m}$ & $50.1(d)$ \\
\hline $8 \mathbf{a}$ & $1.51, \mathrm{~m}$ & 22.6 & $1.58, \mathrm{~m}$ & 22.3 & $1.62, \mathrm{~m}$ & 23.1 \\
\hline $8 b$ & $1.51, \mathrm{~m}$ & $(\mathrm{t})$ & $1.11, \mathrm{~m}$ & (t) & $1.07, \mathrm{~m}$ & $(\mathrm{t})$ \\
\hline 9a & $1.34, \mathrm{~m}$ & $41.5(\mathrm{t})$ & $1.77, \mathrm{~m}$ & $41.0(\mathrm{t})$ & $1.73, \mathrm{~m}$ & $41.8(\mathrm{t})$ \\
\hline $9 b$ & $1.34, \mathrm{~m}$ & & $1.38, \mathrm{~m}$ & & $1.37, \mathrm{~m}$ & \\
\hline 10 & & $73.4(\mathrm{~s})$ & & $72.0(\mathrm{~s})$ & & $73.0(\mathrm{~s})$ \\
\hline 11 & $1.30, \mathrm{~m}$ & $28.7(\mathrm{~d})$ & $1.38, \mathrm{~m}$ & 29.0 (d) & $1.91, \mathrm{~m}$ & $28.1(\mathrm{~d})$ \\
\hline \multirow[t]{2}{*}{12 and 13} & $0.61, \mathrm{~d}(6.8)$ & $21.7(q)$ & $0.66, \mathrm{~d}(6.8)$ & $21.9(\mathrm{q})$ & $0.89, \mathrm{~d}(8.4)$ & $15.6(q)$ \\
\hline & $0.83, \mathrm{~d}(6.8)$ & $15.4(q)$ & $0.89, \mathrm{~d}(6.8)$ & $15.4(q)$ & $0.93, \mathrm{~d}(7.2)$ & $21.8(\mathrm{q})$ \\
\hline 14 & $1.21, \mathrm{~s}$ & $20.5(q)$ & $1.26, \mathrm{~s}$ & $21.0(q)$ & $1.17, \mathrm{~s}$ & $20.1(q)$ \\
\hline 15 & $2.15, \mathrm{~s}$ & $29.8(q)$ & $2.20, \mathrm{~s}$ & 30.1 (q) & $1.18, \mathrm{~d}(6.4)$ & $23.1(q)$ \\
\hline $\mathrm{C}=\mathrm{O}$ & & & & $171.3(\mathrm{~s})$ & & \\
\hline Me & & & $2.04, \mathrm{~s}$ & $21.3(\mathrm{q})$ & & \\
\hline
\end{tabular}

Compounds 3 and $\mathbf{4}$, both isolated as optically active white floc with $[\alpha]_{D}^{20}-17\left(c 0.1, \mathrm{CHCl}_{3}\right)$ and $[\alpha]_{D}^{20}-27$ ( $c 0.1, \mathrm{CHCl}_{3}$ ), respectively, possessed the same molecular formula of $\mathrm{C}_{15} \mathrm{H}_{28} \mathrm{O}_{2}$ based on the positive HRESIMS data at $m / z, 258.2422$ and 258.2428, respectively $\left(\left[\mathrm{M}+\mathrm{NH}_{4}\right]^{+}\right.$, calcd. 258.2428). Their IR peaks at 3398 and $3352 \mathrm{~cm}^{-1}$ suggested the presence of hydroxy groups. Their ${ }^{13} \mathrm{C} N M R$ spectra were similar to those of $\mathbf{1}$, with the exception that the carbonyl carbon at $\delta_{\mathrm{C}} 212.3$ in $\mathbf{1}$ was replaced by hydroxy-bearing methine carbons at $\delta_{\mathrm{C}} 68.6$ and 69.7 in 3 and 4 , respectively. The replacements of the C-4 ketone by secondary hydroxy group were supported by the presence of a quartet at $\delta_{\mathrm{H}} 3.98(1 \mathrm{H}, J=6.4 \mathrm{~Hz}$, assigned to $\mathrm{H}-4)$ of 3 and a doublet of quartet at $\delta_{\mathrm{H}} 4.00(1 \mathrm{H}, J=4.0,6.4 \mathrm{~Hz}$, assigned to $\mathrm{H}-4)$ for 4 in the ${ }^{1} \mathrm{H}$ NMR spectrum, which were confirmed from $\mathrm{HMBC}$ correlations from $\mathrm{H}-4$ to $\mathrm{C}-3$, 
C-5, C-6, and C-15. The structures of $\mathbf{3}$ and $\mathbf{4}$ were thus deduced as epimers of 4,10-dihydroxyoplopanane. The relative configurations of $\mathbf{3}$ and $\mathbf{4}$ were finalized by comparing their spectroscopic features with those of $\mathbf{1}$ and NOE experiment. The large coupling constants of $J_{1,6}=11.6 \mathrm{~Hz}$ in $\mathbf{3 / 4}$ and $\mathbf{3 a} / \mathbf{4 a}$ (acetate of $\mathbf{3 / 4}$, Sect. 3 and Tables 2,3 ) were used to assign $\mathrm{H}-1$ and $\mathrm{H}-6$ as $\alpha$ - and $\beta$ orientation, respectively. Irradiation of H-4 enhanced signals for H-6 and H-11 in 3a (acetate of 3) and 4 allowed $\mathrm{H}-5$ and $\mathrm{H}-7$ as $\alpha$-orientated. Efforts on the determination of the absolute configurations at C-4 in compounds 3 and $\mathbf{4}$ by using Mosher method failed. Therefore the structures of $\mathbf{3}$ and $\mathbf{4}$ were elucidated as depicted and named oplopananol and 4-epi-oplopananol, respectively.

On the basis of NMR, MS, optical rotation data and comparison with literature values, the known sesquiterpenoids were elucidated as oplopanone (5) [11], taiwaninone A (6) [12], T-muurolol (7) [13], $\alpha$-cadinol (8) [14], and clovane-2 $\beta, 9 \alpha$-diol (9) [15]. Naturally occurring oplopanane sesquiterpenoids are rarely reported [16, 17]. Literature searching showed that there were no more than 20 such type sesquiterpenoids reported up to now, distributed among the families of Alismataceae [16, 17], Araliaceae [18], Araceae [19], Asteraceae [20], Chloranthaceae [21], Cyperaceae [22], Magnoliaceae [23],
Meliaceae [24], Schisandraceae [25], Salicaceae [26], and Zingiberaceae [27]. In this study, six oplopananes (1-6), including four new (1-4) and one 3,5-seco-oplopanane (6), were discovered from the rhizomes of $H$. occulta. In addition three new oplopananes $(\mathbf{2 a - 4 a )}$ were also obtained by chemical transformation. These results indicated that $H$. occulta was a rich source of novel natural products.

\section{Experimental Section}

\subsection{General}

Optical rotations were recorded on a 241 polarimeter (Perkin-Elmer). Infrared (IR) spectra were obtained with a FTS 165-IR instrument (Bio-Rad, USA). NMR spectra were acquired on a Varian INOVA-400 FT-NMR spectrometer (USA). HRESIMS were measured on a Bruker APEX II spectrometer. Sephadex LH-20 (Amersham Biosciences) and silica gel (200-300 mesh, Qingdao Haiyang Chemical Co., Ltd) were used for column chromatography (CC), whereas TLC analyses were carried out with glass plates pre-coated with silica gel and the spots were visualized by spraying with $98 \% \mathrm{H}_{2} \mathrm{SO}_{4} / \mathrm{EtOH}$ in $(5 / 95$, v/v)

Table 3 NMR spectroscopic data for compounds $\mathbf{3 a}, \mathbf{4}$, and $\mathbf{4 a}$ in $\mathrm{CDCl}_{3}$

\begin{tabular}{|c|c|c|c|c|c|c|}
\hline \multirow[t]{2}{*}{ Position } & \multicolumn{2}{|l|}{ 3a } & \multicolumn{2}{|l|}{4} & \multicolumn{2}{|l|}{$4 a$} \\
\hline & $\delta_{\mathrm{H}}(J$ in $\mathrm{Hz})$ & $\delta_{\mathrm{C}}$ & $\delta_{\mathrm{H}}(J$ in $\mathrm{Hz})$ & $\delta_{\mathrm{C}}$ & $\delta_{\mathrm{H}}(J$ in $\mathrm{Hz})$ & $\delta_{\mathrm{C}}$ \\
\hline 1 & $1.38, \mathrm{~m}$ & $57.6(d)$ & $1.44, \mathrm{dt}(4.0,12.8)$ & $57.9(d)$ & $1.43, \mathrm{dt}(4.8,11.6)$ & $57.7(d)$ \\
\hline $2 \mathbf{a}$ & $1.70, \mathrm{~m}$ & $25.4(\mathrm{t})$ & $1.77, \mathrm{~m}$ & $26.0(\mathrm{t})$ & $1.74, \mathrm{~m}$ & $26.0(t)$ \\
\hline $2 \mathbf{b}$ & $1.20, \mathrm{~m}$ & & $1.08, \mathrm{~m}$ & & $1.74, \mathrm{~m}$ & \\
\hline 3a & $1.86, \mathrm{~m}$ & $24.4(\mathrm{t})$ & $1.80, \mathrm{~m}$ & $23.6(t)$ & $1.80, \mathrm{~m}$ & $24.2(\mathrm{t})$ \\
\hline $3 \mathbf{b}$ & $1.55, \mathrm{~m}$ & & $1.13, \mathrm{~m}$ & & $1.53, \mathrm{~m}$ & \\
\hline 4 & $4.98, \mathrm{q}(6.4)$ & $73.1(\mathrm{~d})$ & $4.00, \mathrm{dq}(4.0,6.4)$ & $69.7(d)$ & $5.06, \mathrm{~m}$ & 72.7 (d) \\
\hline 5 & $1.38, \mathrm{~m}$ & 46.9 (d) & $2.05, \mathrm{~m}$ & 48.7 (d) & $2.12, \mathrm{~m}$ & $45.2(d)$ \\
\hline 6 & $1.14, \mathrm{~m}$ & $45.1(\mathrm{~d})$ & $0.93, \mathrm{~m}$ & $46.2(\mathrm{~d})$ & $0.98, \mathrm{~m}$ & 45.9 (d) \\
\hline 7 & $1.22, \mathrm{~m}$ & $50.0(\mathrm{~d})$ & $1.21, \mathrm{~m}$ & $50.1(\mathrm{~d})$ & $1.18, \mathrm{~m}$ & 49.9 (d) \\
\hline $8 \mathbf{a}$ & $1.59, \mathrm{~m}$ & $23.3(\mathrm{t})$ & 1.63 , ddt $(3.2,4.0,13.6)$ & $23.4(\mathrm{t})$ & $1.63, \mathrm{dq}(4.0,13.6)$ & $23.2(t)$ \\
\hline $8 b$ & $1.03, \mathrm{dt}(3.6,11.6)$ & & $1.06, \mathrm{~m}$ & & $1.08, \mathrm{~m}$ & \\
\hline 9a & $1.76, \mathrm{dt}(3.6,12.4)$ & $41.9(\mathrm{t})$ & $1.76, \mathrm{~m}$ & $41.9(\mathrm{t})$ & $1.77, \mathrm{~m}$ & $41.9(\mathrm{t})$ \\
\hline $9 b$ & $1.35, \mathrm{~m}$ & & $1.37, \mathrm{dt}(4.0,13.2)$ & & $1.36, \mathrm{dt}(4.0,12.8)$ & \\
\hline 10 & & $73.0(\mathrm{~s})$ & & $73.0(\mathrm{~s})$ & & $73.0(\mathrm{~s})$ \\
\hline 11 & $1.86, \mathrm{~m}$ & $28.2(d)$ & $1.93, \mathrm{~m}(2.4)$ & $28.5(d)$ & $2.09, \mathrm{~m}$ & $28.1(\mathrm{~d})$ \\
\hline \multirow[t]{2}{*}{12 and 13} & $0.72, \mathrm{~d}(7.2)$ & $22.0(q)$ & $0.72, \mathrm{~d}(6.8)$ & $15.7(q)$ & $0.75, \mathrm{~d}(6.8)$ & $15.7(q)$ \\
\hline & $0.90, \mathrm{~d}(6.8)$ & $15.5(\mathrm{q})$ & $0.94, \mathrm{~d}(6.8)$ & $21.9(\mathrm{q})$ & $0.94, \mathrm{~d}(6.8)$ & $22.0(\mathrm{q})$ \\
\hline 14 & $1.12, \mathrm{~s}$ & $19.9(\mathrm{q})$ & $1.14, \mathrm{~s}$ & $20.1(q)$ & $1.14, \mathrm{~s}$ & $20.1(q)$ \\
\hline 15 & $1.19, \mathrm{~d}(6.4)$ & $19.6(q)$ & $1.12, \mathrm{~d}(6.4)$ & $17.0(\mathrm{q})$ & $1.16, \mathrm{~d}(6.4)$ & $13.8(\mathrm{q})$ \\
\hline 16 & & $170.9(\mathrm{~s})$ & & & & $170.6(\mathrm{~s})$ \\
\hline 17 & $2.01, \mathrm{~s}$ & $21.3(\mathrm{q})$ & & & $2.01, \mathrm{~s}$ & $21.5(\mathrm{q})$ \\
\hline
\end{tabular}


followed by heating. All solvents used were analytical grade.

\subsection{Plant Materials}

The rhizomes of H. occulta Lours (Araceae), collected from Guangxi in China, were purchased from Lanzhou Fuxinghou Herbal Medicines Ltd. Co. in February 2007. The materials were identified by Dr. Huan-Yang Qi at Lanzhou Institute of Chemical Physics (LICP), and a voucher specimen (ZY2007H001) was deposited at the herbarium of LICP.

\subsection{Extraction and Isolation}

The air-dried rhizomes $(13.0 \mathrm{~kg})$ of $H$. occulta were powdered and extracted with $88 \%$ ethanol/water $(\mathrm{v} / \mathrm{v})$ at $60{ }^{\circ} \mathrm{C}(12 \mathrm{~h} \times 3)$. After dried in vacuum, the residue $(410 \mathrm{~g})$ was suspended in water $(1.5 \mathrm{~L})$ and applied to a liquid-liquid partitioning against petroleum ether (PE), EtOAc, and $n-\mathrm{BuOH}$ (each $1.0 \mathrm{~L} \times 3$ ) continuously. The dried PE part (257 g) was chromatographed over silica gel $(1.5 \mathrm{~kg}$ ), using gradient PE/acetone ( $\mathrm{v} / \mathrm{v}$, from $80: 1$ to $1: 1$, each about $8.0 \mathrm{~L}$ ) to yield eleven fractions (A1-A11). Fraction A2 $(55 \mathrm{~g})$ was subjected to silica gel CC eluting with $\mathrm{CHCl}_{3} / \mathrm{PE}$ gradient system to afford compound $\mathbf{8}$ $(18.2 \mathrm{mg})$. Fraction A3 $(21 \mathrm{~g})$ was purified over silica gel with $\mathrm{PE} / \mathrm{CHCl}_{3}(\mathrm{v} / \mathrm{v}, 1: 1)$ to afford 7 (4.5 mg). Fraction A5 $(34 \mathrm{~g})$ was fractionated consecutively over silica gel and Sephadex LH-20 $\left(\mathrm{CHCl}_{3} / \mathrm{MeOH}, 1: 1\right.$, v/v $)$ to yield compound 5 (4.5 $\mathrm{mg})$. Fraction A6 (12 g) was chromatographed on a silica gel column eluting with $\mathrm{PE} /$ acetone (v/v, 10:1, 8:1, 5:1, 3:1, and 1:1) to afford compound $1(5.1 \mathrm{mg})$. Fraction A7 $(15 \mathrm{~g})$ was fractionated consecutively over silica gel with $\mathrm{PE} /$ acetone $(8: 1)$ and Sephadex LH-20 $\left(\mathrm{CHCl}_{3} / \mathrm{MeOH}, 1: 1, \mathrm{v} / \mathrm{v}\right)$ to yield 2 $(7.8 \mathrm{mg}), \mathbf{3}(23.1 \mathrm{mg}), \mathbf{4}(17.7 \mathrm{mg}), \mathbf{6}(17.8 \mathrm{mg})$, and $\mathbf{9}$ (2.1 mg).

\subsubsection{7-Epi-oplopanone (1)}

Colorless oil; $[\alpha]_{D}^{20}-54$ (c $0.07, \mathrm{CHCl}_{3}$ ); IR (neat) $v_{\max }$ 3382, 2926, 2857, 1705, 1458, 1383, 1119, 1094, $1027 \mathrm{~cm}^{-1}$; ${ }^{1} \mathrm{H}$ NMR $\left(\mathrm{CDCl}_{3}, 400 \mathrm{MHz}\right)$ and ${ }^{13} \mathrm{C} \mathrm{NMR}$ $\left(\mathrm{CDCl}_{3}, 100 \mathrm{MHz}\right.$ ) (Table 1); HRESIMS $\mathrm{m} / z\left[\mathrm{M}+\mathrm{NH}_{4}\right]^{+}$ 256.2272 (calcd for $\mathrm{C}_{15} \mathrm{H}_{30} \mathrm{O}_{2} \mathrm{~N}, 256.2271$ ).

\subsubsection{5,7-Diepi-2 $\alpha$-hydroxyoplopanone (2)}

Colorless oil; $[\alpha]_{D}^{20}+20\left(c 0.1, \mathrm{CHCl}_{3}\right.$ ); IR (neat) $v_{\max }$ 3392, 2955, 2935, 2871, 1704, 1460, 1380, 1365, 1173, 1121, $1049 \mathrm{~cm}^{-1}$; ${ }^{1} \mathrm{H}$ NMR $\left(\mathrm{CDCl}_{3}, 400 \mathrm{MHz}\right)$ and ${ }^{13} \mathrm{C} \mathrm{NMR}$
$\left(\mathrm{CDCl}_{3}, 100 \mathrm{MHz}\right)$ (Table 2); HRESIMS $\mathrm{m} / \mathrm{z}[\mathrm{M}+\mathrm{Na}]^{+}$ 277.1779 (calcd for $\mathrm{C}_{15} \mathrm{H}_{26} \mathrm{O}_{3} \mathrm{Na}$, 277.1774).

\subsubsection{Oplopananol (3)}

White floc; $[\alpha]_{D}^{20}-17\left(c 0.1, \mathrm{CHCl}_{3}\right)$; IR (neat) $v_{\max } 3398$, 2959, 2933, 1461, 1370, $1129 \mathrm{~cm}^{-1}$; ${ }^{1} \mathrm{H}$ NMR $\left(\mathrm{CDCl}_{3}\right.$, $400 \mathrm{MHz})$ and ${ }^{13} \mathrm{C} \mathrm{NMR}\left(\mathrm{CDCl}_{3}, 100 \mathrm{MHz}\right)$ (Table 2); HRESIMS $\mathrm{m} / \mathrm{z} \quad\left[\mathrm{M}+\mathrm{NH}_{4}\right]^{+} \quad 258.2422$ (calcd for $\left.\mathrm{C}_{15} \mathrm{H}_{32} \mathrm{O}_{2} \mathrm{~N}, 258.2428\right)$.

\subsubsection{4-Epi-oplopananol (4)}

White floc; $[\alpha]_{D}^{20}-27\left(\right.$ c $0.1, \mathrm{CHCl}_{3}$ ); IR (neat) $v_{\max } 3352$, 2956, 2924, 2856, 1571, 1458, 1421, 1365, 1128, 1062, $944 \mathrm{~cm}^{-1} ;{ }^{1} \mathrm{H}$ NMR $\left(\mathrm{CDCl}_{3}, 400 \mathrm{MHz}\right)$ and ${ }^{13} \mathrm{C}$ NMR $\left(\mathrm{CDCl}_{3}, 100 \mathrm{MHz}\right)\left(\right.$ Table 3); HRESIMS $\mathrm{m} / z\left[\mathrm{M}+\mathrm{NH}_{4}\right]^{+}$ 258.2428 (calcd for $\mathrm{C}_{15} \mathrm{H}_{32} \mathrm{O}_{2} \mathrm{~N}, 258.2428$ ).

\subsection{Acetylation of $\mathbf{2}$ to $\mathbf{4}$}

A solution of compounds in a mixture of acetic anhydridepyridine (1:1) were stirred fully and settled at $25{ }^{\circ} \mathrm{C}$ for $12 \mathrm{~h}$. After concentration and storage in vacuo compounds 2a, 3a, and 4a were obtained and identified.

\subsubsection{5,7-Diepi-2 $\alpha$-acetoxyoplopanone (2a)}

Colorless oil; $[\alpha]_{D}^{20}+7\left(c 0.2, \mathrm{CHCl}_{3}\right)$; IR (neat) $v_{\max } 3449$, 2934, 2871, 1734, 1712, 1370, 1247, 1044, $968 \mathrm{~cm}^{-1} ;{ }^{1} \mathrm{H}$ $\mathrm{NMR}\left(\mathrm{CDCl}_{3}, 400 \mathrm{MHz}\right)$ and ${ }^{13} \mathrm{C}$ NMR $\left(\mathrm{CDCl}_{3}\right.$, $100 \mathrm{MHz}$ ) (Table 2); HRESIMS $m / z[\mathrm{M}+\mathrm{Na}]^{+} 319.1883$ (calcd for $\mathrm{C}_{17} \mathrm{H}_{28} \mathrm{O}_{4} \mathrm{Na}, 319.1880$ ).

\subsubsection{4-Acetoxyoplopananol (3a)}

Colorless oil; $[\alpha]_{D}^{20}-41$ (c $\left.0.2, \mathrm{CHCl}_{3}\right) ; \mathrm{UV} \quad\left(\mathrm{CHCl}_{3}\right)$ $\lambda_{\max }(\log \varepsilon) 246$ (1.56) nm; IR (neat) $v_{\max } 3423,2960,2935$, 2892, 1736, 1458, 1375, 1247, 1134, 1033, $930 \mathrm{~cm}^{-1} ;{ }^{1} \mathrm{H}$ NMR $\left(\mathrm{CDCl}_{3}, 400 \mathrm{MHz}\right)$ and ${ }^{13} \mathrm{C}$ NMR $\left(\mathrm{CDCl}_{3}\right.$, $100 \mathrm{MHz}$ ) (Table 3); HRESIMS $m / z$ [M $+\mathrm{Na}]^{+} 305.2091$ (calcd for $\mathrm{C}_{17} \mathrm{H}_{30} \mathrm{O}_{3} \mathrm{Na}, 305.2087$ ).

\subsubsection{4-Epi-acetoxyoplopananol (4a)}

White floc; $[\alpha]_{D}^{20}-10\left(c\right.$ 0.2, $\left.\mathrm{CHCl}_{3}\right) ; \mathrm{UV}\left(\mathrm{CHCl}_{3}\right) \lambda_{\max }(\log$ ع) $246(1.48) \mathrm{nm}$; IR (neat) $v_{\max } 3420,2858,2935,2872$, 1732, 1374, 1248, 1126, 1035, $953 \mathrm{~cm}^{-1}$; ${ }^{1} \mathrm{H}$ NMR $\left(\mathrm{CDCl}_{3}, 400 \mathrm{MHz}\right)$ and ${ }^{13} \mathrm{C} \mathrm{NMR}\left(\mathrm{CDCl}_{3}, 100 \mathrm{MHz}\right)$ (Table 3); HRESIMS $\mathrm{m} / z$ [M $+\mathrm{Na}]^{+} 305.2093$ (calcd for $\mathrm{C}_{17} \mathrm{H}_{30} \mathrm{O}_{3} \mathrm{Na}$, 305.2087). 
Acknowledgments The work was financially supported by the National Nature Science Foundation of China (Nos. 21375136 and 21575150), and the scientific research project of Central Asia Drug Discovery and Development Centre of Chinese Academy of Sciences (No. CAM201404) and the CAS Pioneer Hundred Talents Program.

\section{Compliance with Ethical Standards}

Conflict of Interest The authors declare no competing financial interest.

Open Access This article is distributed under the terms of the Creative Commons Attribution 4.0 International License (http:// creativecommons.org/licenses/by/4.0/), which permits unrestricted use, distribution, and reproduction in any medium, provided you give appropriate credit to the original author(s) and the source, provide a link to the Creative Commons license, and indicate if changes were made.

\section{References}

1. Chinese Pharmacopoeia Commission, Chinese Pharmacopoeia (Part I, 2015 Edition), p33, China Medical Science Press (2015)

2. M. Elbandy, H. Lerche, H. Wagner, M.A. Lacaille-Dubois, Biochem. Syst. Ecol. 32, 1209-1213 (2004)

3. Y.M. Hu, C. Liu, K.W. Cheng, H.H.Y. Sung, L.D. Williams, Z.L. Yang, W.C. Ye, Phytochemistry 69, 2367-2373 (2008)

4. Y.M. Hu, Z.L. Yang, H. Wang, W.C. Ye, Nat. Prod. Res. 23, 1279-1283 (2009)

5. X.Y. Tian, Y. Zhao, S.S. Yu, W.S. Fang, Chem. Biodivers. 7, 984-992 (2010)

6. Y.F. Wang, X.Y. Wang, G.F. Lai, C.H. Lu, S.D. Luo, Chem. Biodivers. 4, 925-931 (2007)

7. X.H. Li, W.J. Zhang, H.Y. Qi, Y.P. Shi, Can. J. Chem. 87, 1218-1221 (2009)
8. R. Wang, W.H. Chen, Y.P. Shi, J. Nat. Prod. 73, 17-21 (2010)

9. R. Wang, L.L. Liu, Y.P. Shi, Helv. Chim. Acta 93, 2081-2085 (2010)

10. J.L. Yang, L.L. Liu, Y.P. Shi, Tetrahedron Lett. 50, 6315-6317 (2009)

11. G.Y. Zhu, G.P. Peng, Nat. Prod. Res. Dev. 14, 85-88 (2002)

12. Y.H. Kuo, C.F. Chyu, Tetrahedron Lett. 44, 7221-7223 (2003)

13. T.V. Sung, L. Kutschabsky, A. Porzel, W. Steglich, G. Adam, Phytochemistry 31, 1659-1661 (1992)

14. W. Herz, K. Watanabe, Phytochemistry 22, 1457-1459 (1983)

15. H. Heymann, Y. Tezuka, T. Kikichi, S. Supriyatna, Chem. Pharm. Bull. 42, 138-146 (1994)

16. G.P. Peng, F.C. Lou, Nat. Prod. Res. Dev. 13, 9-11 (2001)

17. G.Y. Zhu, G.P. Peng, Nat. Prod. Res. Dev. 14, 85-88 (2002)

18. Z.F. Li, Z.H. Wu, G. Chen, Q.H. Zhang, Y.H. Pei, J. Asian Nat. Prod. Res. 11, 715-718 (2009)

19. K.C. Wong, A. Hamid, I.M.S. Eldeen, M.Z. Asmawi, S. Baharuddin, H.S. Abdillahi, J. van Staden, Nat. Prod. Res. 26, 850-858 (2012)

20. L.S. Gan, Z.J. Zhan, S.P. Yang, J.M. Yue, J. Asian Nat. Prod. Res. 8, 589-594 (2006)

21. S. Yang, H, Chen. J. Yue Chin. J. Chem. 30, 1243-1248 (2012)

22. H.B. Xu, Y.B. Ma, X.Y. Huang, C.A. Geng, H. Wang, Y. Zhao, T.H. Yang, X.L. Chen, C.Y. Yang, X.M. Zhang, J.J. Chen, J. Ethnopharmacol. 171, 131-140 (2015)

23. G.J. Zhang, Y.H. Li, J.D. Jiang, S.S. Yu, X.J. Wang, P.Y. Zhuang, Y. Zhang, J. Qu, S.G. Ma, Y. Li, Y.B. Liu, D.Q. Yu, Tetrahedron 70, 4494-4499 (2014)

24. L. Zhang, J.H. Zhang, S.M. Yang, C.H. Tan, H.F. Luo, D.Y. Zhu, J. Asian Nat. Prod. Res. 12, 215-219 (2010)

25. Y. Narukawa, C. Komatsu, R. Yamauchi, S. Shibayama, M. Hachisuka, F. Kiuchi, J. Nat. Med. p 1-7, (2016)

26. W. Wang, Z. Ali, X.C. Li, T.A. Smillie, D.A. Guo, I.A. Khan, Fitoterapia 80, 404-407 (2009)

27. N. Liu, X. Yu, H. Zhao, Y. Zhao, Zhongcaoyao 40, 29-32 (2009) 